$\begin{array}{cc}\text { Programa de Pós-Graduação em Engenharia de Produção - PPGEP } & \\ \text { Laboratório de Qualidade de Vida - LaQVida } & \text { REVISTA BRASILEIRA DE } \\ \text { Universidade Tecnológica Federal do Paraná - UTFPR } & \text { Ponta Grossa - PR - Brasil } \\ \text { v. } 04 \text {, n. 02, jul./dez. 2012, p. } 01-11 & \text { QUALIDADE DE VIDA }\end{array}$

DOI: $10.3895 / \mathrm{S} 2175-08582012000200001$

\title{
A percepção da qualidade de vida em idosos: um estudo exploratório
}

\author{
The perception of quality of life in elderly: an exploratory study
}

\author{
Lara Carvalho Vilela de Lima \\ Universidade de Franca - UNIFRAN - Franca - Brasil \\ laracvilela@hotmail.com \\ Cléria Maria Lobo Bittar \\ Universidade Franca - UNIFRAN - Franca - Brasil \\ cleria@unifran.br
}

\begin{abstract}
Resumo
A presente pesquisa teve como objetivo comparar a percepção de qualidade de vida global de idosos de ambos os sexos que participam de um grupo onde são realizadas atividades de promoção da saúde, com idosos que não participam deste grupo, na cidade de Carneirinho- MG. Trata-se de um estudo quantitativo, composto por uma amostra de 60 idosos, sendo que destes 30 participavam do grupo de atividades em promoção da saúde e outros 30 idosos não participavam. Foram aplicados os questionários WHOQOL-OLD e WHOQOL-BREF. As análises estatísticas foram realizadas a partir do Teste de Mann-Whitney, comparando sempre dois grupos (participantes e não participantes/ masculino e feminino). Adotou-se durante toda a análise $\mathrm{p} \leq 0,05$ como nível de significância, e o programa utilizado foi o SPSS16. Foi constatado que o grupo participante dos encontros da terceira idade apresentou maiores escores nos domínios: autonomia, atividades passadas-presentes-futuras, participação social, morte e morrer e total (WHOQOL-OLD), e nos domínios físico, psíquico, ambiental e total (WHOQOL-BREF). As mulheres do grupo participante apresentaram maiores escores nos domínios autonomia, atividades passadas-presentes-futuras, participação social e total (WHOQOL-OLD), e físico, psíquico, ambiental e total (WHOQOLBREF) em relação as mulheres do grupo não-participante. Os homens do grupo participante apresentaram maiores escores nos domínios: funcionamento sensório, autonomia, participação social e total (WHOQOL-OLD), e em ambiente e total (WHOQOL-BREF) em relação aos homens do grupo não-participante. Entretanto, ao se comparar se houve diferença de percepção de qualidade de vida quanto ao sexos, este estudo não apontou diferenças. Concluiu-se que o grupo participante dos encontros da terceira idade obteve melhores resultados relacionados à percepção de qualidade de vida global e em diversos domínios, quando comparados ao grupo não-participante.
\end{abstract}

Palavras-chave: envelhecimento, promoção da saúde, qualidade de vida.

\begin{abstract}
This research aimed to compare the perception of quality of life overall of elderly people of both sexes who participate in a group where there are activities for health promotion, with older people who do not participate in this group, in the city of Carneirinho - MG. It is a quantitative study, consisting of a sample of 60 elderly people, 30 of them participated in a health promotion group of activities, and other 30 elderly people did not participated. Were applied the questionnaires WHOQOL-OLD and WHOQOL-BREF. Statistical analyzes were carried out using the MannWhitney test, in the comparing of two groups (participants and non-participants/ male and female).
\end{abstract}


It was adopted throughout the analysis $\mathrm{p} \leq 0,05$ significance level, and the program was used SPSS16. It was been found that the participating group presented the greatest scores in the following areas: autonomy, past-present-future activities, social participation, death and dying and total (WHOQOL-OLD), and physical, psychological, environmental and total (WHOQOL-BREF). The women of the participant group had the highest scores in the areas of autonomy, past-presentfuture activities, social participation and total (WHOQOL-OLD), and physical, psychological, environmental and total (WHOQOL-BREF) than the women of the non-participant group. The men of the participant group had the highest scores in the following areas: sensorimotor functioning, independence, social participation and total (WHOQOL-OLD), and environment and total (WHOQOL-BREF) in relation to men in the non- participant group. However, when was compared if there was difference of perception of quality of life for the sexes, this study did not pointed out differences. It was concluded that the group that participated in these meetings of the third age, obtained best results related to the perception of overall quality of life and in a number of areas, when compared to non-participant.

Keywords: aging, health promotion, quality of life.

\section{Introdução}

Diante de tantas mudanças e da evolução da sociedade, valem as palavras de Sauvy (apud BEAUVOIR, 1990, p. 271): "De todos os fenômenos contemporâneos, o menos contestável, o mais certo em sua marcha, o mais fácil de prever com muita antecedência, e, talvez, o de consequências mais pesadas é o envelhecimento da população".

No Brasil, de acordo com Chaimowicz (1997), a partir de 1940 iniciou-se o processo de declínio da mortalidade, que se prolongaria até a década de 1970. Entretanto, somente a partir de 1960, com o declínio da fecundidade em algumas regiões mais desenvolvidas do país, iniciou-se o processo de envelhecimento populacional.

A partir daí, a generalização da redução da fecundidade no Brasil desencadeou o processo de transição da estrutura etária brasileira que, provavelmente, levará a uma nova população quase estável, mas, com um perfil envelhecido e ritmo de crescimento baixo (CARVALHO; WONG, 2008).

Em consequência destas transformações, o número de idosos passou de 3 milhões, em 1960, para 7 milhões em 1975, 17 milhões em 2006 e 20 milhões em 2008 - aumento de quase 600\% em menos de 50 anos (VERAS, 2009). O envelhecimento populacional no Brasil tem revelado um crescimento exponencial, e para o ano de 2025, a projeção mostra que o número de indivíduos com idade igual ou superior a 60 anos de idade será de 32 milhões (PAPALÉO NETTO; YUASO; KITADAI, 2005).

Hoje, o envelhecimento populacional é uma realidade vivenciada no Brasil. O aumento do número de idosos e da expectativa de vida fez com que a sociedade repensasse o seu ponto de vista sobre a velhice e os desafios que são lançados para uma vida melhor. Para Carvalho e Wong (2008), com o aumento da população idosa, a sociedade necessita investir na atual geração de crianças, nas áreas de seguridade social, saúde e educação, pois, não se trata de garantir apenas a melhoria da qualidade de vida dessas gerações, mas o equilíbrio de toda a sociedade.

O prolongamento da vida é uma aspiração de qualquer sociedade, mas, só pode ser considerado como uma real conquista na medida em que se agregue qualidade aos anos adicionais de vida (VERAS, 2009).

O termo qualidade de vida começou a ser usado como um conceito nos Estados Unidos após a segunda guerra mundial, com o objetivo de caracterizar a vida das pessoas com base na aquisição de bens materiais (FERRAZ, 1998 apud BUONFIGLIO; CUNHA; AKERMAN, 2005). Pouco depois, os economistas e sociólogos buscaram encontrar índices que permitiam avaliar a qualidade de vida dos indivíduos e das sociedades (ALBUQUERQUE, 2003). Posteriormente, o termo passou a se referir a uma série de definições dentro das mais diversas áreas, tais como a filosofia, a 
enfermagem, a psicologia e a medicina; entretanto este conceito se encontra na sua infância e precisa amadurecer, mas tem pela frente uma longa história, pois veio para ficar (BUONFIGLIO; CUNHA; AKERMAN, 2005).

No contexto de atenção à saúde, de acordo com Paschoal (2006), chegou-se a conclusão de que vida longa não era necessariamente boa vida. $O$ interesse médico no construto qualidade de vida foi estimulado pelo sucesso em se prolongar a vida e pela compreensão de que isso pode ser um benefício equivocado, pois os pacientes querem viver, e não meramente sobreviver.

Apesar de haver um consenso sobre a importância de avaliar a qualidade de vida, seu conceito ainda gera complexidade. Portanto, a definição proposta pela Organização Mundial de Saúde, através do Grupo WHOQOL, é a que melhor traduz a abrangência do construto qualidade de vida. O Grupo WHOQOL definiu qualidade de vida como "[...] a percepção do indivíduo de sua posição na vida, no contexto de sua cultura e no sistema de valores em que vive e em relação a suas expectativas, seus padrões e suas preocupações" (WHOQOL Group, 1995 apud FLECK et al., 2008 , p. 25). Esta definição considera o conceito de qualidade de vida como sendo bastante amplo, e incorpora, de forma complexa, a saúde física, o estado psicológico, o nível de independência, as relações sociais, as crenças pessoais e a relação com aspectos significativos do meio ambiente (FLECK et al., 2008).

O desafio que se propõe aos indivíduos e à sociedade é conseguir uma sobrevida cada vez maior, com uma qualidade de vida cada vez melhor, para que os anos vividos em idade avançada sejam plenos de significado e dignidade (PASCHOAL, 2006). Pensando assim, é preciso dar suporte ao crescente número de velhos, priorizando uma visão ampla, centrada na promoção da saúde com o objetivo de se garantir ou restabelecer a qualidade de vida nesta faixa etária.

Para Veras e Caldas (2004), a proposta para os que já são idosos é a de promover a saúde por meio da manutenção ou recuperação da autonomia e da independência, postergando ao máximo o início das doenças que na maior parte das vezes são crônicas. De acordo com Veras (2006), o pressuposto principal é a garantia da inclusão social e da participação cidadã do idoso. Há, portanto, necessidade de uma articulação política consistente no sentido de incluir na agenda social as reivindicações desse segmento populacional.

A promoção da saúde visa garantir o envelhecimento saudável ou bem-sucedido, definido por Carvalhaes Neto (2005, p. 9) como “[...] aquele com baixa suscetibilidade a doenças e elevada capacidade funcional (física e cognitiva), acompanhado de uma postura ativa perante a vida e a sociedade". Segundo Ramos (2005), o envelhecimento saudável passa a ser a resultante da interação multidimensional entre saúde física, saúde mental, independência na vida diária, integração social, suporte familiar e independência econômica.

Tomando como exemplo os campos da promoção da saúde do idoso, encontra-se nos grupos da terceira idade uma forma de realizar ações que vão em busca do envelhecimento saudável. Pena e Santo (2006) explicam que o aumento da expectativa e a qualidade de vida das pessoas idosas estão associados não somente a evolução da tecnologia e medicina, mas, também, à vivência dos idosos em grupos. Esta vivência transcende as atividades físicas e de lazer, e os idosos são incentivados a se expressarem, vivenciando vários sentimentos, e emoções.

Diante do exposto e vivenciando o envelhecimento da população de Carneirinho - MG foi desenvolvido na cidade um projeto com um grupo de idosos denominado Envelhecimento Saudável, cuja finalidade é realizar semanalmente atividades de promoção, prevenção e educação na área da saúde, com a participação voluntária de uma equipe interdisciplinar e de colaboradores, com o objetivo de melhorar a qualidade de vida dos idosos do local.

Neste contexto, o presente estudo tem por objetivo comparar a percepção de qualidade de vida global de idosos de ambos os sexos que participam de um grupo onde são realizadas atividades de promoção da saúde, com idosos que não participam deste grupo, na cidade de Carneirinho- MG.

\section{Métodos}

A presente pesquisa caracteriza-se como um estudo exploratório de caráter quantitativo, cujo 
corte adotado foi transversal.

O estudo foi realizado na cidade de Carneirinho- MG, cuja população cadastrada no Sistema de Informação de Atenção Básica- SIAB é de 4.368 pessoas (SIAB, 2009). Destas, 582 tinham idade igual ou acima de 60 anos de idade, 294 homens e 288 mulheres. De acordo com esses números, $13,324 \%$ da população de Carneirinho eram constituídas por idosos, ou seja, pessoas com 60 ou mais anos de idade.

Foram utilizados nesta pesquisa os questionários WHOQOL-OLD e WHOQOL-BREF, instrumentos específicos para avaliação de qualidade de vida, principalmente no que diz respeito aos idosos, no caso do questionário WHOQOL-OLD.

O WHOQOL-BREF consta de 26 questões, sendo duas questões gerais de qualidade de vida e as demais 24 representam cada uma das 24 facetas que compõem o instrumento original (WHOQOL100). O questionário abreviado é composto por quatro domínios: físico, psicológico, relações sociais e meio ambiente. Já o questionário WHOQOL-OLD tem como objetivo acessar itens adicionais, relevantes e complementares aos já alocados nos instrumentos genéricos de medição de qualidade de vida (WHOQOL-100 e BREF), sendo composto pelos domínios: funcionamento dos sentidos, autonomia, atividades passadas- presentes -futuras, participação social, morte e morrer e intimidade (FLECK et al., 2008).

\subsection{Local e população do estudo}

O estudo foi realizado com idosos (idade igual ou acima de 60 anos de idade) cadastrados no SIAB e na Associação Alegre da Melhor Idade de Carneirinho (AAMIC), onde foi desenvolvido o projeto Envelhecimento Saudável. Existem em torno de 40 pessoas que frequentam regularmente os encontros da terceira idade realizados na AAMIC, número que varia no decorrer do ano. Destas pessoas, apenas uma não tem 60 anos e foi excluída da pesquisa. A amostra foi constituída por 30 idosos, pois estes atenderam aos critérios de inclusão. Os demais idosos que participam dos encontros semanais do grupo da terceira idade não participaram da pesquisa, por não atenderem aos critérios de inclusão, descritos na sequência.

Portanto, participaram da pesquisa 60 sujeitos: 30 do grupo da terceira idade denominado grupo participante (GP) cuja amostra foi constituída por 20 pessoas do sexo feminino e 10 do sexo masculino, e 30 que não faziam parte deste, denominado grupo não participante (GNP), cuja amostra manteve a mesma distribuição por sexos, de forma a manter a mesma proporção de sujeitos do GP.

Os idosos do GP foram aqueles que atenderam aos critérios de inclusão adotados: sujeitos com idade igual ou superior a 60 anos, cadastrados tanto no SIAB como na AAMIC; que participassem dos encontros semanais realizados no grupo da terceira idade há pelo menos seis meses; que fossem independentes para realizar as atividades de vida diárias, plenamente capazes e em plena capacidade funcional e cognitiva.

Os 30 sujeitos que participaram do GNP foram selecionados por frequentarem o ambulatório de saúde, constituindo uma amostra por conveniência. Os critérios de inclusão foram: ter 60 anos ou mais, ser cadastrado no SIAB, sem nenhuma doença incapacitante que pudesse atrapalhar a participação no grupo, em plena capacidade funcional e cognitiva e que nunca tivesse frequentado os encontros da terceira idade.

Os questionários WHOQOL-BREF e WHOQOL-OLD foram aplicados aos 60 idosos, individualmente, entre junho e agosto de 2009, em suas respectivas residências. Os encontros foram agendados pessoalmente com cada idoso, no qual foi marcado um encontro para que eles pudessem responder aos instrumentos acima. Os questionários foram aplicados por uma pesquisadora. As perguntas foram lidas pela pesquisadora e respondidas pelos idosos, devido a maior parte dos idosos incluídos na pesquisa serem analfabetos.

Além disso, todos os idosos consentiram em participar da pesquisa dando suas anuências no Termo de Consentimento Livre e Esclarecido (TCLE). Esta pesquisa foi desenvolvida no Programa de Mestrado em Promoção de Saúde, da Universidade de Franca, sendo submetido ao Comitê de 
Ética da referida instituição, conforme estabelecido na Resolução 196/96 do Conselho Nacional de Saúde (CNS), recebendo aprovação final em 24 de junho de 2010, sob o protocolo de n. 007/09.

\subsection{Delineamento da pesquisa}

Foram realizadas as análises estatísticas descritivas dos questionários WHOQOL-OLD e BREF, a partir do Teste de Mann-Whitney, comparando sempre dois grupos (participantes e não participantes/ masculino e feminino). As análises completas dos questionários estão divididas em relação grupo/sexo/mulheres/homens entre os dois grupos. Adotou-se durante toda a análise $\mathrm{p} \leq$ 0,05 como nível de significância, e o programa utilizado foi o SPSS16.

\section{Resultados}

Na Tabela 1 foram descritos os resultados encontrados através da análise dos questionários WHOQOL-OLD e WHOQOL-BREF entre os dois grupos de idosos: GP e GNP através do teste de Mann-Whitney.

Os resultados mostraram que houve diferenças significativas entre os grupos de idosos, sendo que o GP apresentou maiores escores nos domínios: autonomia, atividades passadaspresentes-futuras, participação social, morte e morrer e total-old (WHOQOL-OLD). No questionário WHOQOL-BREF os domínios: físico, psíquico, ambiental e total-bref do GP obtiveram valores significativamente superiores nas respostas quando comparados com o GNP.

Tabela 1- Comparação entre grupo participante (GP) e não- participante (GNP):

\section{WHOQOL-OLD e WHOQOL-BREF}

\begin{tabular}{lcc}
\hline Facetas & $\begin{array}{c}\text { Houve diferenças significativas } \\
\text { entre os dois grupos (GP e } \\
\text { WHOQO) }\end{array}$ & $\begin{array}{c}\text { Não houve diferenças } \\
\text { significativas entre os dois } \\
\text { grupos (GP e GNP) }\end{array}$ \\
\hline $\begin{array}{l}\text { Funcionamento Sensório } \\
\text { Autonomia }\end{array}$ & $(\mathrm{p}<0,001)$ & \\
Atividades PPF & $(\mathrm{p}=0,009)$ & \\
Participação Social & $(\mathrm{p}<0,001)$ & \\
Morte e Morrer & $(\mathrm{p}=0,04)$ & $(\mathrm{p}=0,16)$ \\
Intimidade & $(\mathrm{p}<0,001)$ & \\
\hline TOTAL-OLD & & \\
\hline Facetas & $(\mathrm{p}=0,004)$ & \\
WHOQOL-BREF & $(\mathrm{p}=0,002)$ & \\
\hline Físico & $(\mathrm{p}=0,34)$ \\
Psíquico & $(\mathrm{p}<0,001)$ & \\
Social & & \\
Ambiental & & \\
\hline TOTAL- BREF & & \\
\hline
\end{tabular}

Fonte: Autoria própria (2009).

Ao comparar o GP e GNP em relação ao sexo, verificou-se que não houve diferença significativa entre homens e mulheres dos dois grupos. Todavia, na presente pesquisa, houve diferenças significativas entre participantes e não participantes do mesmo sexo.

Na tabela 2 foram descritos os resultados encontrados a partir da análise entre os grupos de idosas e os questionários WHOQOL-OLD e BREF, no qual foi utilizado o Teste de Mann-Whitney.

Os resultados mostraram que o GP apresentou valores significativamente superiores no questionário WHOQOL-OLD para os domínios: autonomia, atividades passadas-presentes-futuras, participação social e total-old. Valores mais altos foram encontrados no questionário WHOQOLBREF nos domínios físico, psíquico, ambiental e total-bref para idosas do GP em relação às idosas do GNP. 
Tabela 2 - Comparação entre grupo participante (GP) e não-participante (GNP) do sexo feminino: WHOQOL-OLD e WHOQOL-BREF

\begin{tabular}{lcc}
\hline $\begin{array}{l}\text { Facetas } \\
\text { WHOQOL-OLD }\end{array}$ & $\begin{array}{c}\text { Houve diferenças significativas } \\
\text { entre os dois grupos (GP e } \\
\text { GNP) }\end{array}$ & $\begin{array}{c}\text { Não houve diferenças } \\
\text { significativas entre os dois } \\
\text { grupos (GP e GNP) }\end{array}$ \\
\hline $\begin{array}{l}\text { Funcionamento Sensório } \\
\text { Autonomia } \\
\text { Atividades PPF }\end{array}$ & $(\mathrm{p}=0,55)$ \\
$\begin{array}{l}\text { Participação Social } \\
\text { Morte e Morrer }\end{array}$ & $(\mathrm{p}=0,001)$ & \\
Intimidade & $(\mathrm{p}<0,001)$ & \\
\hline TOTAL - OLD & & $(\mathrm{p}=0,15)$ \\
\hline Facetas & $(\mathrm{p}=0,001)$ & $(\mathrm{p}=0,28)$ \\
WHOQOL-BREF & & \\
\hline Físico & & \\
Psíquico & $(\mathrm{p}=0,005)$ & $(\mathrm{p}=0,68)$ \\
Social & $(\mathrm{p}=0,004)$ & \\
Ambiental & $(\mathrm{p}=0,001)$ & \\
\hline TOTAL - BREF & $(\mathrm{p}<0,001)$ & \\
\hline
\end{tabular}

Fonte: Autoria própria (2009).

$\mathrm{Na}$ Tabela 3 foram descritos os resultados encontrados a partir da análise do teste MannWhitney entre os dois grupos do sexo masculino e entre os dois questionários.

Observou-se que o GP apresentou maiores escores no questionário WHOQOL-OLD nos domínios: funcionamento sensorial, autonomia, participação social e total-old, em relação ao GNP. No questionário WHOQOL-BREF o GP apresentou maiores escores em relação ao GNP para os domínios: ambiental e total-bref.

Tabela 3 - Comparação entre grupo participante (GP) e não-participante (GNP) do sexo masculino:

\begin{tabular}{lcc} 
Facetas & WHOQOL-OLD W WOQOL-BREF & \\
WHOQOL-OLD & $\begin{array}{c}\text { Houve diferenças significativas } \\
\text { entre os dois grupos (GP e } \\
\text { GNP) }\end{array}$ & $\begin{array}{c}\text { Não houve diferenças } \\
\text { significativas entre os dois } \\
\text { grupos (GP e GNP) }\end{array}$ \\
\hline $\begin{array}{l}\text { Funcionamento Sensório } \\
\begin{array}{l}\text { Autonomia } \\
\text { Atividades PPF }\end{array}\end{array}$ & \\
$\begin{array}{l}\text { Participação Social } \\
\text { Morte e Morrer }\end{array}$ & $(\mathrm{p}=0,04)$ & $(\mathrm{p}=0,05)$ \\
Intimidade & $(\mathrm{p}=0,05)$ & $(\mathrm{p}=0,25)$ \\
\hline TOTAL - OLD & & $(\mathrm{p}=0,48)$ \\
\hline Facetas & $(\mathrm{p}=0,04)$ & \\
WHOQOL-BREF & & $(\mathrm{p}=0,35)$ \\
\hline $\begin{array}{l}\text { Físico } \\
\text { Psíquico }\end{array}$ & & $(\mathrm{p}=0,32)$ \\
Social & & $(\mathrm{p}=0,32)$ \\
Ambiental & $(\mathrm{p}=0,01)$ & \\
\hline TOTAL - BREF & $(\mathrm{p}=0,05)$ & \\
\hline
\end{tabular}

Fonte: Autoria própria (2009).

\section{Discussão}

A tabela 1 descreveu a comparação da percepção de qualidade de vida de idosos do GP com idosos do GNP. Os resultados mostram que os idosos do GP apresentaram maiores escores no questionário WHOQOL-OLD para os domínios: autonomia, atividades passadas-presentes-futuras, participação social, morte e morrer e total-old. Nos domínios funcionamento sensorial e intimidade não houve diferenças entre os dois grupos, portanto, houve diferenças significativas entre os grupos de idosos. 
No WHOQOL-BREF os domínios: físico, psíquico, ambiental e total-bref do GP obtiveram valores significativamente superiores nas respostas quando comparados com o GNP. No domínio social não houve diferenças significativas entre os grupos (GP e GNP).

Miranda e Banhato (2008) afirmaram que idosos que participaram ativamente de algum grupo perceberam uma melhor qualidade de vida e apresentaram maiores escores em todas as facetas do questionário WHOQOL-OLD se comparados àqueles que não participaram regularmente de nenhuma atividade em grupo.

Ao descrever a qualidade de vida de idosos que participaram de um grupo de convivência, Serbim e Figueiredo (2011) encontraram que a faceta do questionário WHOQOL-OLD que mais contribuiu na qualidade de vida dos sujeitos foi funcionamento sensório, seguida de morte e morrer e participação social.

No presente estudo, o domínio físico apresentou maior escore para o GP em relação ao GNP. Segundo Pereira et al. (2006), a influência do domínio físico na qualidade de vida global ressalta a importância de se considerar a capacidade funcional como importante fator de impacto na qualidade de vida em idosos. Além disso, a autonomia e independência também têm sido associadas à qualidade de vida na senescência.

Pereira et al. (2006) ressaltaram que se deve considerar o ambiente físico em que o idoso está inserido, uma vez que o domínio ambiental também apresenta grande influência na qualidade de vida destes idosos. Dados semelhantes foram encontrados no presente estudo, no qual o GP obteve maiores escores no domínio ambiental quando comparados com os idosos do GNP.

Em relação ao domínio psicológico, a presente pesquisa apontou que os idosos do GP apresentaram maiores escores em relação aos idosos do GNP. Ao comparar idosos que participaram de um projeto na Universidade Aberta da Terceira Idade com idosos não engajados em atividades sociais, Carneiro et al. (2007) encontraram diferenças significativas para o domínio psicológico para o grupo de idosos que participaram das atividades realizadas no projeto. Destacou-se, que as diversas atividades realizadas em grupos, dentre elas as de cunho social, proporcionam possíveis benefícios sobre os fatores psicológicos, os quais refletem em uma melhor qualidade de vida dos idosos.

Magesky, Modesto e Torres (2009) observaram após uma intervenção psicossocial realizada com grupo de idosos institucionalizados, uma melhora no relacionamento interpessoal e na desnaturalização de crenças e mitos que interferem na qualidade de vida dos idosos. Portanto, através desta intervenção foi possível repensar e reinventar práticas psicológicas levando em consideração o contexto ao qual o sujeito está imerso, contribuindo assim para a compreensão do processo de envelhecimento.

Os resultados da presente pesquisa mostraram que a percepção de qualidade de vida global dos idosos do GP em relação ao GNP foi maior, demonstrado nos escores do domínio total OLD e total BREF (Tabela 1).

Castro et al. (2007) relataram o ganho de qualidade de vida para idosos que participaram de um programa de atividades para a terceira idade e apontaram a importância do incentivo para que idosos participem dessas e de outras atividades a fim de prevenir o isolamento e a imobilização que podem ocorrer no processo de envelhecimento.

Programas que envolvem o trabalho educacional permanente, com a abordagem de uma equipe interdisciplinar, compostos por atividades físicas, sociais e culturais, melhoram a percepção dos fatores relacionados aos domínios psicológico e ambiental, bem como a percepção de qualidade de viga global dos idosos (CASTRO et al., 2007).

O domínio social não apresentou diferença entre os grupos deste estudo, o qual trouxe uma reflexão. Como a cidade é muito pequena e os idosos estão quase sempre em situação de envolvimento social ou familiar, é provável que este entorno social seja o responsável pela não diferença neste domínio, para ambos os grupos.

Resultado semelhante foi observado por Castro et al. (2007). Na pesquisa não foi encontrada melhora significativa no domínio social na avaliação de um grupo de idosos que participaram de programas para a terceira idade. Segundo Pereira et al. (2006), embora o domínio social não tenha 
demonstrado contribuição significativa para a qualidade de vida dos idosos avaliados em seu estudo, há que se considerar a inserção social pelo qual passam os idosos, de forma a minimizar os efeitos dessas transformações decorrentes da idade na qualidade de vida desses sujeitos.

$\mathrm{Na}$ comparação entre o GP e GNP em relação ao sexo, verificou-se que não houve diferenças significativas entre homens e mulheres dos dois grupos. Entretanto, é preciso considerar as peculiaridades existentes na vida de homens e mulheres para garantir a equidade entre gêneros. Como destacaram Pereira et al. (2006), é importante reconhecer a heterogeneidade da população idosa no que diz respeito ao sexo e à idade, além da variância individual, quando se avalia qualidade de vida.

Todavia, neste estudo, houve diferenças entre idosos participantes e não participantes do sexo feminino, conforme a Tabela 2.

Ao comparar o GP e GNP do sexo feminino e o WHOQOL-OLD, verificou-se que o GP obteve valores superiores em relação ao GNP para os domínios: autonomia, atividades passadaspresentes-futuras, participação social e total-old. Os domínios funcionamento sensório, morte e morrer e intimidade não apresentaram diferenças entre as idosas dos dois grupos.

Valores mais altos também foram encontrados no WHOQOL-BREF nos domínios físico, psíquico, ambiental e total-bref para idosas do GP em relação às idosas do GNP. O domínio social não apresentou diferença entre os grupos.

Em relação ao domínio físico, os resultados do presente estudo confirmaram o estudo de Vilela Júnior et al. (2010), no qual este domínio foi o que mais contribuiu para a percepção da qualidade de vida de mulheres idosas fisicamente ativas. Tal resultado permite ressaltar evidências positivas entre a atividade física regular e qualidade de vida.

Os grupos da terceira idade são espaços que oferecem mudanças positivas na vida das idosas. Em estudo realizado com mulheres, Bulsing et al. (2007) afirmaram que os grupos de convivência da terceira idade não são apenas um passatempo, como alguns acreditam, mas têm muita importância para as idosas, possibilitando-lhes a ressocialização, o aumento do seu grupo de amizades e a melhora da autoestima. Proporcionando, um novo sentido para a vida daquela mulher que antes se sentia sozinha e sem muitas expectativas.

A tabela 3 descreveu os dados relativos à comparação da percepção de qualidade de vida dos idosos do sexo masculino dos dois grupos. Em relação ao questionário WHOQOL-OLD, verificouse que o GP apresentou maiores escores em relação ao GNP nos domínios: funcionamento sensorial, autonomia, participação social e total-old. Nos domínios atividades passadas-presentesfuturas, morte e morrer e intimidade não houve diferenças entre os grupos.

No WHOQOL-BREF o GP apresentou maiores escores em relação ao GNP nos domínios: ambiental e total-bref. Em relação aos domínios físico, psíquico e social não houve diferenças entre idosos dos dois grupos.

Os motivos para a participação em grupos da terceira idade estão de acordo com os interesses dos gêneros. As mulheres têm mais consciência da necessidade de realizar atividades físicas para obter uma melhor qualidade de vida, o homem que passou praticamente a vida toda fora de casa, com a aposentadoria, passa mais tempo em casa. Assim, a ocupação por homens nos espaços da terceira idade, torna-se uma maneira de preencher o lugar das atividades que os mesmos realizavam anteriormente. Portanto, essas práticas sociais são experiências significativas e contribuem muito qualitativamente no processo de envelhecimento (COUTINHO; ACOSTA, 2009).

Os grupos de promoção de saúde ao atuarem no campo comunitário abrem possibilidades para as ciências da saúde e do homem, horizontes que vão além do ato de combater as doenças dos indivíduos. Estes deverão acrescentar à sua tarefa, a preocupação com a sua própria identidade, a busca do mais alto grau possível de saúde física, social e mental, tanto para si quanto para a sociedade (SANTOS et al., 2006).

Como pode ser visto neste estudo, a percepção de qualidade de vida global dos idosos do GP, tanto dos homens quanto das mulheres, foi melhor em relação aos idosos que não participaram das atividades no grupo da terceira idade. 
Resultados semelhantes foram encontrados por Almeida et al. (2010), nos quais os idosos que participaram de um grupo de convivência apresentaram melhor qualidade de vida e menor ocorrência de depressão em relação aos idosos que não participaram de grupos.

Segundo Tahan e Carvalho (2010), a ideia atual de saúde está associada a sentir-se bem e feliz, trocar experiências e vivências com o outro, o que proporcionam de fato os grupos. Além disto, os grupos são fortes auxiliares na formação de uma rede de apoio social, na integração da comunidade com os serviços de saúde, na melhora da percepção da saúde e consequentemente da qualidade de vida.

Estes grupos permitem aos homens e mulheres, por meio do resgate da solidariedade, o processo de superação física e psicológica de um nível individual para grupal, e deste para um mais amplo, que é o social (SANTOS et al., 2006).

Para Rizzolli e Surdi (2010), a participação de idosos em grupos contribuiu com mudanças favoráveis em diversos aspectos em sua vida, tais como: na conquista de novas amizades, na melhora da saúde e na sua valorização, os quais refletiram em uma melhor qualidade de vida dos mesmos.

Diante de questões tão complexas quanto o envelhecimento, a qualidade de vida e a promoção da saúde, novos estudos são necessários para um maior aprofundamento e compreensão de ações que colaborem para que não só os idosos, mas todas as faixas etárias tenham suas necessidades levadas em consideração na criação das práticas de saúde (TAHAN; CARVALHO, 2010). Em relação às práticas de saúde, o trabalho com grupos de idosos mostrou ser uma estratégia importante na busca da promoção da saúde na senescência.

\section{Conclusão}

O envelhecimento populacional é um fenômeno vivenciado no Brasil, entretanto, esta vivência ocorre de diferentes formas em locais de dimensões distintas. Dessa forma, torna-se importante o conhecimento sobre a percepção de qualidade de vida deste contingente populacional.

Os resultados encontrados no presente estudo mostraram uma melhor percepção de qualidade de vida dos idosos pertencentes ao GP. Dos 30 idosos que participaram de um grupo onde são realizadas atividades de promoção da saúde 26 apresentaram avaliação boa ou muito boa de sua qualidade de vida quando comparados com idosos que não participaram das atividades grupais, sendo que deste grupo apenas 18 idosos relataram boa ou muito boa qualidade de vida.

Os idosos do GP apresentaram maiores escores em relação aos idosos do GNP nos domínios autonomia, atividades passadas-presentes-futuras, participação social, morte e morrer e total-old no questionário WHOQOL-OLD e físico, psíquico, ambiental e total-bref relativos ao WHOQOLBREF.

Não houve diferenças entre a percepção de qualidade de vida entre homens e mulheres. Entretanto, as mulheres do GP apresentaram maiores escores nos domínios autonomia, atividades passadas-presentes-futuras, participação social e total-old no questionário WHOQOL-OLD e nos domínios físico, psíquico, ambiental e total-bref no questionário WHOQOL-BREF. Em relação aos idosos do sexo masculino, o GP apresentou maiores escores nos domínios funcionamento sensorial, autonomia, participação social e total-old no WHOQOL-OLD e para os domínios ambiental e totalbref no questionário WHOQOL-BREF.

Ainda que o domínio social do questionário WHOQOL-BREF não apresentasse diferenças entre os dois grupos de idosos, isto sugere que a dimensão populacional da cidade e a facilidade de deslocamento das pessoas idosas devem ser levadas em consideração, na medida em que permite aos idosos estarem em diversos ambientes sociais, proporcionando a manutenção de vínculos afetivos e sociais, ainda que não estivessem frequentando os encontros da terceira idade.

Conclui-se que as atividades realizadas nos grupos da terceira idade sugerem formas positivas de proporcionar aos idosos as condições ensejadas nas premissas da promoção da saúde, refletindo em uma melhor percepção de qualidade de vida destes idosos. 


\section{Referências}

ALBUQUERQUE, S. M. R. L. Qualidade de vida do idoso: a assistência domiciliar faz a diferença? São Paulo: Casa do Psicólogo, 2003.

ALMEIDA, E. A. et al. Comparação da qualidade de vida entre idosos que participam e idosos que não participam de grupos de convivência na cidade de Itabira- MG. Revista Brasileira de Geriatria e Gerontologia, Rio de Janeiro, v. 13, n. 3, p. 435-443, 2010.

BEAUVOIR, S. A velhice. Tradução de Maria Helena Franco Martins. Rio de Janeiro: Nova Fronteira, 1990.

BUONFIGLIO, F.; CUNHA, E.; AKERMAN, M. Qualidade de vida: conceitos, dimensões e aplicações. São Paulo: Mídia Alternativa, 2005.

BULSING, F. L. et al. A influência dos grupos de convivência sobre a autoestima das mulheres idosas do município de Santa-Cruz do Sul - RS. Revista Brasileira de Ciências do Envelhecimento Humano, Passo Fundo, v. 4, n. 1, p. 11-17, jan./jun. 2007.

CARNEIRO, R. S. et al. Qualidade de vida, apoio social e depressão em idosos: relação com habilidades sociais. Psicologia: Reflexão e Crítica, v. 20, n. 2, p. 229-237, 2007.

CARVALHAES NETO, N. Envelhecimento bem-sucedido e envelhecimento com fragilidade. In: RAMOS, L. R.; TONIOLO NETO, J. Geriatria e gerontologia: guias de medicina ambulatorial e hospitalar Unifesp - Escola Paulista de Medicina. Barueri: Manole, 2005. cap. 2, p. 9-25.

CARVALHO, J. A. M.; WONG, L. R. A transição da estrutura etária da população brasileira na primeira metade do século XXI. Cadernos de Saúde Pública, Rio de Janeiro, v. 24, n. 3, p. 597605 , mar. 2008.

CASTRO, P. C. et al. Influência da Universidade Aberta da Terceira Idade (UATI) e do Programa de Revitalização (REVT) sobre a qualidade de vida de adultos de meia-idade e idosos. Revista Brasileira de Fisioterapia, São Carlos, v. 11, n. 6, p. 461-467, nov./dez. 2007.

CHAIMOWICZ, F. A saúde dos idosos brasileiros às vésperas do século XXI: problemas, projeções e alternativas. Revista de Saúde Pública, São Paulo, v. 31, n. 2, p. 184-200, abr. 1997.

COUTINHO, R. X.; ACOSTA, M. A. de F. Ambientes masculinos da terceira idade. Ciência \& Saúde Coletiva, v. 14, n. 4, p. 1111-1118, 2009.

FLECK, M. P. de A. et al. Problemas conceituais em qualidade de vida. In: (Org.). A avaliação de qualidade de vida: guia para profissionais da saúde. Porto Alegre: Artmed, 2008. cap. 1, p. 19-28.

MAGESKY, A. M.; MODESTO, J. L.; TORRES, L. C. A. Intervenção Psicossocial com um grupo de idosos institucionalizados. Revista Saúde e Pesquisa, v. 2, n. 2, p. 217-224, mai./ago. 2009.

MIRANDA, L. C.; BANHATO, E. F. C. Qualidade de vida na terceira idade: a influência da participação em grupos. Psicologia em Pesquisa, Juiz de Fora, v. 2, n. 1, p. 69-80, jan./jun. 2008.

PAPALÉO NETTO, M.; YUASO, D. R.; KITADAI, F. T. Longevidade: desafio no terceiro milênio. O Mundo da Saúde, São Paulo, v. 29, n. 4, p. 594-607, out./dez. 2005. 
PASCHOAL, S. M. P. Qualidade de vida na velhice. In: FREITAS, E. V. et al. Tratado de geriatria e gerontologia. 2. ed. Rio de Janeiro: Guanabara Koogan, 2006. cap. 14, p. 147-153.

PENA, F. B.; SANTO, F. H. do E. O movimento das emoções na vida dos idosos: um estudo com um grupo da terceira idade. Revista Eletrônica de Enfermagem, v. 8, n. 1, p. 17-24, 2006. Disponível em: <http://www.fen.ufg.br/revista/ revista8_1/original_02.htm>. Acesso em: 6 ago. 2011.

PEREIRA, R. J. et al. Contribuição dos domínios físico, social, psicológico e ambiental para a qualidade de vida global de idosos. Revista de Psiquiatria do Rio Grande do Sul, v. 28, n. 1, p. 27-38, jan./abr. 2006.

RAMOS, L. R. A mudança de paradigma na saúde e o conceito de capacidade funcional. In: RAMOS, L. R.; TONIOLO NETO, J. Geriatria e gerontologia: guias de medicina ambulatorial e hospitalar Unifesp - Escola Paulista de Medicina. Barueri: Manole, 2005. cap. 1, p. 1-7.

RIZZOLLI, D; SURDI, A. C. Percepção dos idosos sobre grupos de terceira idade. Revista Brasileira de Geriatria e Gerontologia, Rio de Janeiro, v. 13, n. 2, p. 225-233, ago. 2010.

SANTOS, L. M. et al. Grupos de promoção à saúde no desenvolvimento da autonomia, condições de vida e saúde. Revista de Saúde Pública, v. 40, n. 2, p. 346-352, 2006.

SERBIM, A. K.; FIGUEIREDO, A. E. P. L. Qualidade de vida de idosos em um grupo de convivência. Scientia Medica, Porto Alegre, v. 21, n. 4, p. 166-172, 2011.

SIAB: Sistema de informação de atenção básica: Carneirinho/MG. 2009. Disponível em: <http://tabnet.datasus.gov.br/cgi/deftohtm.exe?siab/cnv/ SIABSMG. def. Acesso em: 10 set. 2009.

TAHAN, J.; CARVALHO, A. C. D. de. Reflexões de Idosos Participantes de Grupos de Promoção de Saúde Acerca do Envelhecimento e da Qualidade de Vida. Saúde e Sociedade, São Paulo, v. 19, n. 4, p. 878-888, 2010.

VERAS, R. P.; CALDAS, C. P. Promovendo a saúde e a cidadania do idoso: o movimento das universidades da terceira idade. Ciência \& Saúde Coletiva, v. 9, n. 2, p. 423-432, 2004.

VERAS, R. P. Envelhecimento humano: ações de promoção à saúde e prevenção de doenças. In: FREITAS, E. V. et al. Tratado de geriatria e gerontologia. 2. ed. Rio de Janeiro: Guanabara Koogan, 2006. cap. 13, p. 140-146.

VERAS, R. Envelhecimento populacional contemporâneo: demandas, desafios e inovações. Revista de Saúde Pública, v. 43, n. 3, p. 548-554, 2009.

VILELA JÚNIOR, G. de B. et al. Qualidade de vida e capacidade de equilíbrio durante o processo de envelhecimento de mulheres praticantes de atividade física. Revista Brasileira de Qualidade de vida, Ponta Grossa, v. 2, n. 2, p. 18-25, jul./dez. 2010. 